\title{
Basiliximab as Induction in Kidney Transplantation: Are There any Real-Life Advantages?
}

\author{
Mitra Pa , Rahim MA ${ }^{\mathrm{b}}$, Samdani TS ${ }^{\mathrm{c}}$, Haque $\mathrm{WMM}^{\mathrm{d}}$, Iqbal Se, Mansur MA ${ }^{\mathrm{f}}$
}

\begin{abstract}
Background: Basiliximab is an interleukin-2 receptor antagonist used as induction therapy in kidney transplantation and is believed to reduce acute rejection episode (ARE). Our aims were to compare the impact of basiliximab induction therapy with no induction therapy on incidence of ARE, time requirement for serum creatinine (S.Cr) to normalize after transplantation, initial post-transplant hospital stay, infection in immediate post-transplant period, chronic allograft injury and graft survival at 1 and 3 years.
\end{abstract}

Methods: We retrospectively reviewed the medical records of patients who had undergone living related donor kidney transplantation in a tertiary care hospital of a developing country between July 2004 and June 2014. We selected patients who received calcineurin inhibitors, mycophenolate and prednisolone to classify as no induction therapy ( $n=50$; group 1, receiving prednisolone, mycophenolate and cyclosporine as maintenance therapy) and induction therapy with basiliximab ( $n=61$; group 2, receiving prednisolone, mycophenolate and tacrolimus as maintenance therapy).

Results: Among the 111 study subjects, only two had experienced ARE (one from each group, $p=0.889$ ). Patients who received basiliximab had a shorter mean hospital stay (11.4 \pm 3.3 versus $13.7 \pm 5.0$ days, $p=0.005)$ and shorter mean duration for normalization of $S . C r$ (4.7 \pm 2.3 versus $7.3 \pm 5.6$ days, $p=0.002)$ after transplantation. There was no significant difference in incidence of infection in immediate post-transplant period $(p=0.134)$. One year graft survival rate was better in those who received basiliximab $(98.2 \%$ versus $89.4 \%, p=0.010)$ but there was no significant difference at 3 years $(79 \%$ versus $74 \%, p=0.549)$. Overall incidence of chronic allograft injury was less with basiliximab (11.5\% versus $36 \%, p=0.002)$ induction.

Conclusions: Induction therapy with basiliximab was associated with shorter mean hospital stay, early renal function recovery, better 1 year graft survival and less overall incidence of chronic allograft injury. We have encountered minimum ARE to comment on benefit of basiliximab on ARE.

Key words: Basiliximab, induction therapy, kidney transplantation.

(BIRDEM Med J 2017; 7(2): 90-94)

Author Information

a. Dr. Palash Mitra, Assistant Registrar, Nephrology and Dialysis, BIRDEM General Hospital, Dhaka, Bangladesh.

b. Dr. Muhammad Abdur Rahim, Assistant Professor, Nephrology and Dialysis, BIRDEM General Hospital, Dhaka, Bangladesh.

c. Dr.Tasrina Shamnaz Samdani, Assistant Professor, Medicine, Delta Medical College and Hospital, Dhaka, Bangladesh.

d. Dr. Wasim Md. Mohosin U1 Haque, Associate Professor, Nephrology and Dialysis, BIRDEM General Hospital, Dhaka, Bangladesh.

e. Dr. Sarwar Iqbal, Professor, Nephrology and Dialysis, BIRDEM General Hospital, Dhaka, Bangladesh.

f. Dr. Md. Abul Mansur, Professor, Nephrology and Dialysis, BIRDEM General Hospital and Director, Transplant Unit, BADAS, Dhaka, Bangladesh.

Address of Correspondence:Dr. Palash Mitra, Assistant Registrar, Nephrology and Dialysis, BIRDEM General Hospital, Dhaka, Bangladesh. Email: palash_mtr@yahoo.com

Received: October 28, 2016

Accepted: February 28, 2017

\section{Introduction}

Chronic kidney disease (CKD) is a global public health problem. It is the $9^{\text {th }}$ leading cause of death in the United States. ${ }^{1}$ End stage renal disease (ESRD) patients need renal replacement therapy (RRT) for survival. Globally 1.4 million patients are receiving RRT and its frequency is growing by about $8 \%$ per year. $^{2}$ According to the UK Renal Registry $17^{\text {th }}$ Annual Report, 56,940 adult patients were receiving RRT in the UK on $31^{\text {st }}$ December $2013 .^{3}$ Renal transplantation is the optimum form of RRT, it has an unequivocal superiority over dialysis considering life expectancy, quality of life and cost. ${ }^{4-7}$

First successful kidney transplantation was done in 1954 by Joseph Murray's team between identical twins. ${ }^{8}$ In the early era of transplantation, total body irradiation and 6-marcaptopurine were used for immuno- 
suppression. Unacceptable rate of mortality, high rate of rejection and unsatisfying graft survival warranted the development of new immunosuppressive agents over decades. Introduction of cyclosporine in $1980 \mathrm{~s}$ revolutionized the scenario followed by introduction of tacrolimus and different mono- or poly-clonal antibody induction agents over the next 3 decades. ${ }^{9}$ With the availability of antibody based induction agents, immunosuppressive protocols are now individualized on the basis of recipient characteristics (high or low immunological risk group (Figure 1). ${ }^{10}$ To minimize the side effects of steroid and to avoid the cyclosporine induced nephropathy, "steroid avoidance" and "calcineurin inhibitors (CNI) minimization" protocols are evolved. In our center, the first 50 cases received cyclosporine based immunosuppressive therapy [cyclosporine, mycophenolate mofetil (MMF) and steroid] and currently we are using basiliximab induction with triple immunosuppressive regimen (tacrolimus, MMF and steroid).

Basiliximab is an interleukin-2 receptor (IL-2R) antagonist used as induction therapy in kidney transplantation and is believed to reduce acute rejection episodes (ARE). Our aims were to compare the impact of basiliximab induction therapy with no induction therapy on incidence of ARE, time requirement for serum creatinine (S.Cr) to normalize after transplantation, initial post-transplant hospital stay, infection in immediate post-transplant period, chronic allograft injury and graft survival at 1 and 3 years.

\section{Methods}

We retrospectively reviewed the medical records of patients who had undergone living related donor kidney transplantation in Bangladesh Institute of Research and Reahibilitation in Diabetes, Endocrine and Metabolic Disorders (BIRDEM) General Hospital, Dhaka, Bangladesh, between July 2004 and June 2014. We selected patients who received calcineurin inhibitors (cyclosporine or tacrolimus), MMF and prednisolone to classify as no induction therapy $(\mathrm{n}=50$; Group 1 , with oral prednisolone, MMF and cyclosporine as maintenance immunosuppression) and induction therapy with basiliximab $(n=61$; Group 2, with oral prednisolone, MMF and tacrolimus as maintenance immunosuppression). Collected records were then compared between group 1 and group 2 in respect of ARE, time required for normalization of serum creatinine (S.cr) level, infection and 1 and 3 year graft survival.

$\begin{array}{lr}\text { Induction agent } \\ \text { No induction }<\text { Basiliximab }<\text { Alemtuzumab }<\text { Anti-thymocyte globulin } \\ \text { Lower risk } \\ \text { Zero HLA mismatch } \\ \text { Live donor } & \text { Increased tt of HLA mismatche } \\ \text { Caucasian ethnicity } & \text { Younger recipient and older donor age } \\ \text { Low panel reactive antibody } & \text { African-American ethnicity } \\ \text { Absence of donor specific antibody } & \text { High panel reactive antibody } \\ \text { Blood group compatibility } & \text { Presence of donor specific antibody } \\ \text { Immediate graft function } & \text { Blood group incompatability } \\ \text { Short cold ischemia time } & \text { Delayed onset of graft function } \\ \text { First transplant } & \text { Long cold ischemia time } \\ \end{array}$

Figure 1. Induction therapy choice based on risk assessment [Adopted from Hardinger et al. (2012), Transplant International] ${ }^{10}$ 


\section{Results}

Total number of patients were 111 with a male predominance (male:female $=2.3: 1$ ) and mean age was $37.5 \pm 10.6$ years without any significant deference between the groups (Table-I). Patients who received basiliximab had a shorter mean hospital stay and shorter mean duration for normalization of S.Cr after transplantation (Table-II). There was no significant difference in incidence of infection in immediate posttransplant period $(\mathrm{p}=0.134)$. One year graft survival rate was better in those who received basiliximab but there was no significant difference at 3 years (Figure 2). Among the total of 111 study subjects only two had experienced ARE (one from each group, $p=0.889$ ). Overall incidence of chronic allograft injury was less with basiliximab ( $11.5 \%$ versus $36 \%, p=0.002)$ induction.
Table I. Demography of Group 1 and Group 2 patients

\begin{tabular}{lccc} 
& Group 1* & Group 2** & p-value \\
\hline Number of patients & 50 & 61 & \\
Male :Female & $1.9: 1$ & $2.6: 1$ & 0.4839 \\
Mean age (years) & $36.8 \pm 10.8$ & $38.0 \pm 10.4$ & 0.5534 \\
\hline
\end{tabular}

* Group 1: No induction; ** Group 2: Induction with Basiliximab

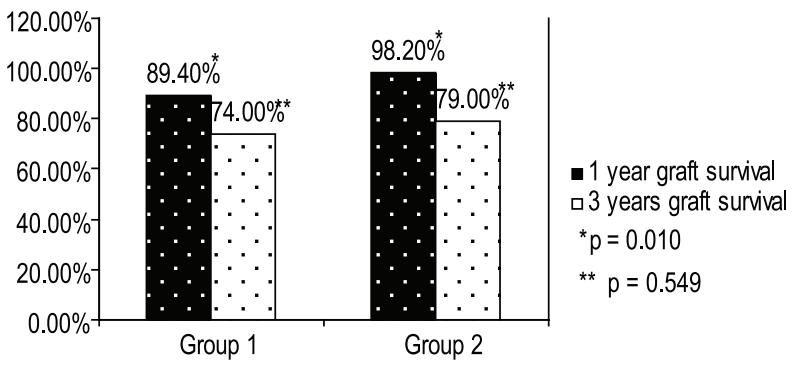

Figure 2. Comparison of graft survival between Group 1 and Group 2 patients

Table II. Comparison between Group 1 and Group 2 patients

\begin{tabular}{lccc} 
& Group 1 & Group 2 & p value \\
\hline Mean duration for normalization of S. Cr (days) & $7.3 \pm 5.6$ & $4.7 \pm 2.3$ & 0.002 \\
Mean S. Cr during discharge (mg/dl) & $1.4 \pm 0.91$ & $1.3 \pm 0.6$ & 0.258 \\
Incidence of infection in immediate post-transplant period & 17 & 13 & 0.134 \\
Mean post-transplant hospital stay (days) & $13.7 \pm 5.0$ & $11.4 \pm 3.3$ & 0.005 \\
\hline
\end{tabular}

\section{Discussion}

In current time, the world is divided into two regions, developed world and developing world, depending on economic and technological advancement between countries. About $80 \%$ of the world population resides in developing world who owns only about $25 \%$ of the total wealth and only 0.8 to $4 \%$ of fund is used in healthcare sector in comparison to 10 to $15 \%$ in developed countries. ${ }^{11}$

Bangladesh is a developing country of South-Asia, with a population burden of 160 million (approx.). Nephrology is relatively a juvenile branch of medical science in our country. In most instances when a renal physician attends a CKD patient, he is already in stage 4 or 5 CKD or developed ESRD with other complications of the disease. First renal transplantation was done in our country in 1982. Due to lack of facilities we could not perform few tests like donor specific antibody (DSA) or panel reactive antibody (PRA) but the other essential tests could be performed with high efficiency.

In our centre, BIRDEM General Hospital, first renal transplantation was performed in 2004. Initially we used the triple drug regime with steroid, cyclosporine and MMF. The optimum immunosuppressive therapy was not always possible to provide due to non-availability of all the usable drugs and high cost of them. Since December 2009, we have introduced tacrolimus (FK506) instead of cyclosporine and the induction therapy with injection basiliximab in patients with $25 \%$ to $50 \%$ match in humen leukocyte angena typing. Though we have marked these patients as immunologically low risk 
group, considering their history of blood transfusion and lack of sensitivity information regarding DSA or PRA, their actual immunological risk could not be defined.

We have found significantly better 1 year graft survival in our patients with basiliximab induction group with shorter time requirement for normalization of serum creatinine in post-transplant period and shorter posttransplant mean hospital stay. But there was no significant difference in graft survival at 3 years of transplantation. No comments could be made regarding acute rejection episode as the incidence was least in both groups.

Jurewicz WA et al. from UK reported that they have followed up 232 renal transplant recipients in the University Hospital of Wales, Cardiff for 6 years and found that tacrolimus-based immunosuppression was associated with superior short and long-term renal function than cyclosporine based immunosuppression. ${ }^{12}$ Similar interpretation was made by Webster AC et al. ${ }^{13}$ in their report, where they reviewed 123 reports from 30 studies that involved 4102 kidney transplant recipients patients. Vardhan $\mathrm{H}$ et al. studied 296 kidney transplant recipients in India and found that acute rejection was significantly less in patients with basiliximab induction compared to those without induction as well as more favourable short and long term graft outcome in basiliximab induction group. ${ }^{14}$ On the other hand Koch $\mathrm{M}$ et al. reported that graft and patient survival was equivalent, 10 years after transplantation, with or without basiliximab induction therapy. ${ }^{15}$

In the studies, comparing the no induction group with basiliximab induction group of renal transplant recipients, the maintenance immunosuppressive protocol was cyclosporine based and the results reflected a less acute rejection episode with favourable short and long term graft survival effects. ${ }^{14}$ The same benefits have been achieved by using tacrolimus instead of cyclosporine. ${ }^{12,13}$ As we are using basiliximab induction in conjunction with tacrolimus based maintenance immunosuppressive agents, it is not clear whether the favourable results are either due to basiliximab or tacrolimus.

It is important to ascertain whether the favourable outcomes like shorter mean hospital stay, early renal function recovery, better 1 year graft survival and less overall incidence of chronic allograft injury, as we are observing in our transplant patients, is due to tacrolimus or basiliximab. In a developing country like ours, the extra economic burden of induction therapy must be justified. There is still scope of further study to clarify the role of tacrolimus and basiliximab individually regarding acute rejection, short and long term graft survival in our patient groups and also to justify the role of basiliximab induction therapy in our patients.

Declaration: This research paper was presented in the 14th Congress of the Asian Society of Transplantation (CAST), Singapore, 2015.

\section{References}

1. Albright A, Burrows NR, Jordan R, Williams DE. The Kidney Disease Initiative and the Division of Diabetes Translation at the Centers for Disease Control and Prevention. American Journal of Kidney Diseases: AJKD 2009;53(3 Suppl 3): S121-25.

2. Schieppati A, Remuzzi G. Chronic renal diseases as a public health problem: epidemiology, social, and economic implications. Kidney International 2005; 98 (Suppl): S7-10.

3. Rao A, Casula A, Castledine C. UK Renal Registry 17th Annual Report: Chapter 2 UK Renal Replacement Therapy Prevalence in 2013: National and Centre-specific Analyses. Nephron 2015;129 (Suppl 1):31-56.

4. Wolfe RA, Ashby VB, Milford EL, Ojo AO, Ettenger RE, Agodoa LYC, et al. Comparison of Mortality in All Patients on Dialysis, Patients on Dialysis Awaiting Transplantation, and Recipients of a First Cadaveric Transplant. NEJM 1999;341(23):1725-30.

5. Oniscu GC, Brown H, Forsythe JL. Impact of cadaveric renal transplantation on survival in patients listed for transplantation. JASN 2005;16(6):1859-65.

6. Maglakelidze N, Pantsulaia T, Tchokhonelidze I, Managadze L, Chkhotua A. Assessment of health-related quality of life in renal transplant recipients and dialysis patients. Transplant Proc 2011;43(1):376-79.

7. Fisher R, Gould D, Wainwright S, Fallon M. Quality of life after renal transplantation. Journal of Clinical Nursing 1998; 7(6):553-63.

8. Merrill JP, Murray JE, Harrison J, Guild WR. Successful homotransplantation of the human kidney between identical twins. JAMA 1956;160(4):277-82.

9. Zand MS. Seminars in Dialysis. 2005;18(6): 511-19.

10. Hardinger KL, Brennan DC, Klein CL. Selection of induction therapy in kidney transplantation. Transplant International 2013;26(7):662-72

11. Rizvi SA, Naqvi SA, Hussain Z, Hashmi A, Akhtar F, Hussain $\mathrm{M}$, et al. Renal transplantation in developing countries. Kidney International 2003; 83(Suppl.): S96-100. 
12. Jurewicz WA. Tacrolimus versus cyclosporin immunosuppression: long-term outcome in renal transplantation. Nephrology Dialysis Transplantation: 2003;18(Suppl 1): i7-11.

13. Webster A, Woodroffe RC, Taylor RS, Chapman JR, Craig JC. Tacrolimus versus cyclosporin as primary immunosuppression for kidney transplant recipients. The Cochrane Database of Systematic Reviews 2005(4): Cd003961.
14. Vardhan H, Prasad N, Jaiswal A, Yadav B, Kumar S, Bhadauria D, et al. Outcomes of living donor renal transplant recipients with and without basiliximab induction: A longterm follow-up study. Indian Journal of Transplantation 2014; $8(2): 44-50$.

15. Koch M, Becker T, Lueck R, Neipp M, Klempnauer J, Nashan B. Basiliximab induction therapy in kidney transplantation: Benefits for long term allograft function after 10 years? Biologics: Targets \& Therapy 2009;3:51-56. 